\title{
A. GuÉnOCHE
}

\section{Counting and selecting at random bipartite graphs with fixed degrees}

Revue française d'automatique, d'informatique et de recherche opérationnelle. Recherche opérationnelle, tome 24, nº 1 (1990), p. 1-14.

$<$ http://www.numdam.org/item?id=RO_1990_24_1_1_0>

(C) AFCET, 1990, tous droits réservés.

L'accès aux archives de la revue « Revue française d'automatique, d'informatique et de recherche opérationnelle. Recherche opérationnelle » implique l'accord avec les conditions générales d'utilisation (http://www.numdam.org/ legal.php). Toute utilisation commerciale ou impression systématique est constitutive d'une infraction pénale. Toute copie ou impression de ce fichier doit contenir la présente mention de copyright.

\section{Numdam}

Article numérisé dans le cadre du programme

Numérisation de documents anciens mathématiques

http://www.numdam.org/ 


\title{
COUNTING AND SELECTING AT RANDOM BIPARTITE GRAPHS WITH FIXED DEGREES ( $\left.{ }^{*}\right)$
}

\author{
by A. GuÉNOCHe $\left({ }^{1}\right)$
}

\begin{abstract}
We first recall the bijective mapping between bipartite simple graphs with specified degrees and 0/1 arrays with fixed sums of rows and columns, and we count these graphs, using an enumerative procedure. Tables that contain the numbers of such graphs with at most 10 edges are given. Then we put a total order on this set, and provide an algorithm to build the graph that have a given rank. If this rank is selected at random, uniformely or not, we get a random bipartite simple graph.

Keywords : Combinatorics; enumeration; random graphs.

Résumé. - Après avoir rappelé la correspondance bijective entre les graphes simples bipartis de degrés fixés et les tableaux en $0 / 1$ de totaux marginaux fixés, nous dénombrons ces graphes, $a$ l'aide d'une procédure d'énumération. Ensuite on munit cet ensemble d'un ordre total afin de présenter un algorithme qui construit le graphe de rang donné pour cet ordre. Si ce rang est tiré au hasard (uniformément), on obtient un graphe aléatoire (équiprobable). Nous donnons en annexe les tables de dénombrement des graphes bipartis de degrés fixés jusqu'à 10 arêtes, pour toute séquence de degrés.
\end{abstract}

Mots clés : Algorithmes Combinatoires; énumération; graphes aléatoires.

\section{INTRODUCTION}

Frequently computer programs that permit graph editing are used for theorical research and/or teaching graph theory. These programs are more efficient if they present random generation functions for graphs with given properties. After many authors, we propose an algorithm of this family to build bipartite graphs with a specified degree sequence. To build a simple graph with given degrees the "natural" algorithm, that selects at random edges until saturation of the degrees, may cancel if one unsaturated vertex is left, or if it there remains some vertices already linked with preceding edges.

(*) Received November 1987.

( $\left.{ }^{1}\right)$ GRTC-CNRS, 31, Ch.-J. Aiguier, 13402 Marseille Cedex 9.

Recherche opérationnelle/Operations Research, 0399-0559/90/01 1 14/\$3.40 (c) AFCET-Gauthier-Villars 
If it fails, the procedure is restarted until success. We will call this algorithm "with rejection".

N. C. Wormald [4] proposes such an algorithm to build a bipartite simple graph with $n+m$ vertices having specified degrees. The two degree sequences are partitions $A=\left(a_{1}, \ldots, a_{n}\right)$ and $B=\left(b_{1}, \ldots, b_{m}\right)$ of the number of edges $N=\sum_{i=1, \ldots, n} a_{i}=\sum_{i=1, \ldots, m} b_{i}$. He notices that a $0 / 1$ array with $n$ rows and $m$ columns, that presents $A$ as the sums of the rows and $B$ as the sums of the columns, is a code for these graphs. So he proposes to select at random a composition of $N$ with type $\left(a_{1}, \ldots, a_{n}\right)$, that is a word $\left(x_{1}, \ldots, x_{N}\right)$ of length $N$ over the alphabet $[n]=\{1,2, \ldots, n\}$, such that the letter $i$ occurs $a_{i}$ times. Therefore, the $b_{1}$ first letters of this word are the row indices of the first column, the $b_{2}$ following letters the index of the second column and so on. This procedure may fail if the same letter $i$ appears many times in one of the $b_{j}$ sequences of consecutive letters.

\section{Exemple 1}

$A=$ (2211), $B=(222), N=6$. If the selected random word is $(2,3,1,4,1,2)$ we will obtain the following array and graph:

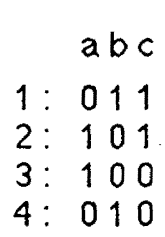

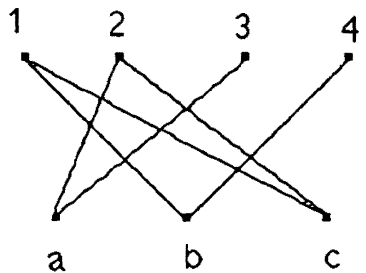

But if the random word is $(1,1,2,3,2,4)$ we do not get a simple graph since the same vertex in the first part is linked twice to the first vertex of the second part.

N. C. Wormald proves that his iterative procedure succeeds and he gives an upper bound for the number of trials.

In this text we propose an algorithm that selects at first shot, without rejection, a $0 / 1$ array with specified sums of rows and columns, that is a bipartite simple graph with fixed degrees. It is based on the number of such arrays that we study in the first part. We give a recurrent function and a procedure to count them. In the second part we put a total order on this set and we build the array that have rank $R$ for this order. If $R$ is uniformely selected, we obtain a random graph with a uniform probability. 


\section{NUMBER OF 0/1 ARRAYS WITH FIXED SUMS OF ROWS AND COLUMNS}

Let $T\left(a_{1}, \ldots, a_{N r} / b_{1}, \ldots, b_{N c}\right)$, also noted $T(A / B)$, be the number of $0 / 1$ arrays with $N r$ rows and $N c$ columns such that sums of rows are $a_{1} \leqq a_{2} \leqq \ldots a_{N r}$, and sums of columns are $b_{1} \leqq b_{2} \leqq \ldots b_{N c}$. The orders of the rows and the colums are arbitrary. We choose the one that corresponds to the classical notation of partitions of $N$ in lexicographical order.

Since T (A/B) is a binary array (resp. a simple graph) we must have $a_{N r} \leqq N c$ and also $b_{N c} \leqq N r$ (resp. the maximum degree of a vertex in a part cannot exceed the cardinality of the other part). So any couple of partitions of $N(A$ and $B$ ) cannot lead to a $0 / 1$ array. In this case we put $T(A / B)=0$.

LEMMA

$$
T\left(a_{1}, \ldots, a_{N r} / b_{1}, \ldots, b_{N c-1}, N r\right)=T\left(a_{1}-1, \ldots, a_{N r}-1 / b_{1}, \ldots, b_{N c-1}\right) .
$$

If $\mathrm{b}_{\mathrm{Nc}}=\mathrm{Nr}$ the last column contains only 1's. We do not change the number of arrays taking off this column and subtracting 1 to all the parts of $A$.

More generally, we are going to count these arrays using a recursive formula based on the number of ways anabling to remove as much as the number of ones in the last column, substracting one unity to some parts of $A$.

THEOREM

$$
T(A / B)=\sum_{\mathscr{C}\left(b_{N_{c}}, N_{r}\right)} T\left(A^{\prime} / B^{\prime}\right)
$$

with $\mathscr{C}(K, L)=\{$ subsets with $K$ elements in a set with $L$ elements $\}$, $B^{\prime}=\left(b_{1}, \ldots, b_{N c-1}\right)$ and $A^{\prime}$ is a partition of $N-b_{N c}$ obtained from $A$, removing one unity to the parts selected in an element of $\mathscr{C}\left(b_{N c}, N r\right)$.

In this formula, the last column is considered as the characteristic vector of a set with $b_{N c}$ elements in the set of the $N r$ rows. The preceding lemma is the situation where $\mathscr{C}(K, L)$ is reduced to a single element since $K=L$.

\section{1. Algorithm to compute $T(A / B)$}

The principle of the algorithm is to remove the greatest part $B M=b_{N c}$ from $B$-we obtain a partition $B^{\prime}=\left(b_{1}, \ldots, b_{N c-1}\right)$ - and to subtract one unity to $B M$ parts of $A$. To each way to choose these $B M$ parts among the $N r$ parts of $A$, corresponds a partition $A^{\prime}$ of $N-B M$. 
We can enumerate characteristic vectors $C=(c(i), i=1, \ldots, N r)$ that indicate the $B M$ elements in a set with $N r$ elements, and each partition $A^{\prime}$ is equal to $A-C$. After that we have to reorder the parts different from 0 .

Exemple 2: $A=\left(\begin{array}{lll}1 & 1 & 1\end{array}\right), B=\left(\begin{array}{ll}1 & 22\end{array}\right)$

\begin{tabular}{|c|c|c|c|}
\hline 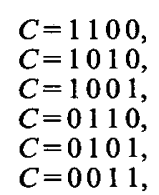 & $\begin{aligned} A^{\prime} & =\left(\begin{array}{ll}1 & 2\end{array}\right), \\
A^{\prime} & =\left(\begin{array}{ll}1 & 2\end{array}\right) \\
A^{\prime} & =\left(\begin{array}{lll}1 & 1 & 1\end{array}\right) \\
A^{\prime} & =\left(\begin{array}{ll}1 & 2\end{array}\right) \\
A^{\prime} & =\left(\begin{array}{lll}1 & 1 & 1\end{array}\right) \\
A^{\prime} & =\left(\begin{array}{lll}1 & 1 & 1\end{array}\right)\end{aligned}$ & $B^{\prime}=(12)$ & $\begin{aligned} T\left(A^{\prime} / B^{\prime}\right) & =1 \\
& =1 \\
& =3 \\
& =1 \\
& =3 \\
& =3\end{aligned}$ \\
\hline & & & $=12$ \\
\hline
\end{tabular}

This method provides a great lot of identical vectors $A^{\prime}$, and the procedure is too long. We get the partition (12) when we reduce two parts equal to 1 . In the same way we get the partition (111) when we reduce one part equal to 1 and the part equal to 2 . Both happen 3 times. To obtain only once each partition $A^{\prime}$, we consider the notation $A=\left[d_{1}, d_{2}, \ldots, d_{p}\right]$ in which $p=a_{N r}$ and $d_{k}$ is the number of parts equal to $k$. It is sufficient now to enumerate the compositions $C=\left(c_{1}, c_{2}, \ldots, c_{p}\right)$ of $B M$ into $p$ parts bounded by $\left(d_{1}, d_{2}, \ldots, d_{p}\right)$ and to weight $T\left(A^{\prime} / B^{\prime}\right)$ with $q_{c}=\prod_{i=1, \ldots, p}\left(d_{i} \backslash c_{i}\right)$ where $\left(d_{i} \backslash c_{i}\right)$ is the binomial coefficient.

Taking back the previous exemple : $A=\left[\begin{array}{ll}31 \\ 1\end{array}, B=\left(\begin{array}{ll}122\end{array}\right)\right.$,

$\begin{array}{llll}C=(20), & A^{\prime}=[11], & q_{c}=(3 \backslash 2), & B^{\prime}=\left(\begin{array}{ll}12 \\ A^{\prime}\end{array}\right) \\ C=(11), & \left.A^{\prime}=\square\right], & q_{c}=(3 \backslash 1), & B^{\prime}=\left(\begin{array}{ll}12\end{array}\right)\end{array}$

Enumeration of compositions of $M$ in $P$ parts bounded with $D(1), \ldots, D(P)$

The enumeration algorithm for compositions of $M$ in $P$ parts given by Nijenhuis et Wilf [3], based on their bijective mapping with subsets with $M$ elements in a set with $M+P-1$ elements, cannot be easily extended to the bounded parts. So we elaborate the following algorithm that gives, while Flag $=0$, the next composition. Initially Flag $:=1$ and the first composition is computed.

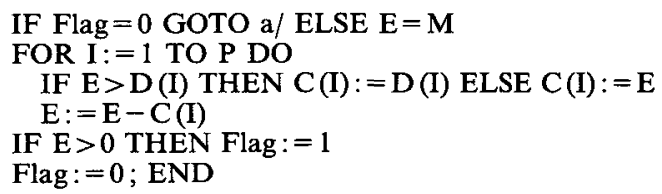


a/ We seek to the last part that can be reduced, and we give the greatest possible values to the following parts.

$\mathrm{EE}:=\mathrm{C}(\mathrm{P})$

FOR $\mathrm{I}:=\mathrm{P}-1$ TO 1 AND $\mathrm{C}(\mathrm{I})>0$ DO

$\mathrm{EE}:=\mathrm{EE}+1 ; \mathrm{C}(\mathrm{I}):=\mathrm{C}(\mathrm{I})-1: \mathrm{E}:=\mathrm{EE}$

FOR $\mathrm{J}:=1+1$ TO $P$ DO

IF E $>$ D (J) THEN C (J) : = D (J) ELSE C (J) $:=\mathrm{E}$

$\mathrm{E}:=\mathrm{E}-\mathrm{C}(\mathrm{J})$

IF $\mathrm{E}=0$ THEN END ELSE EE $:=\mathrm{EE}+\mathrm{C}(\mathrm{I})$

Flag: $=1$; END

\subsection{Tabulation}

To realise efficiently the algorithm that builds the given rank array, we must have tables that give for any couple of partitions of $K \leqq N$, the number of arrays that present these partitions as the sums of rows and columns. If we store these partitions themselves, we will occupy an excessive amount of memory. So we define a characteristic function for the partitions of $N$. Starting with a rank $R$, this function computes a partition $A$ according to lexicographical order.

Assuming C. Berge's [1] notations, let

$\mathscr{P}_{n}=\{$ partitions of $n\}$,

$\mathscr{P}_{n, h}=\{$ partitions of $n$ such that the smallest part is equal to $h\}$,

$P_{n}$ and $P_{n, h}$ their numbers of elements. We have the obvious relation:

$$
P_{n, h}=P_{n-1, h-1}-P_{n-h, h-1} \quad(h>1)
$$

that permits to compute the values stored into an array $P$ with two indices $n$ and $h$, starting with $P_{n, 1}=P_{n-1}, P_{n, n}=1$ and $\Sigma_{h} P_{n, h}=P_{n}$. The rank $R$ of a partition $(A(I), I=1, N P)$ of $N$ in $N P$ parts is given by the following procedure.

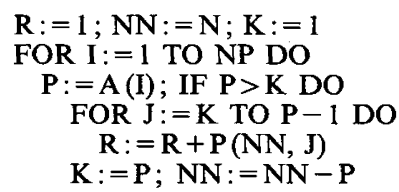

Reciprocally, to the rank $R$ corresponds a partition of $N,(A(I), I=1, N P)$ that we obtain counting the number of partitions that have a rank smaller than $R$. Thus we add the number of partitions of which the smallest part is $1,2, \ldots, I+1$, until we reach or overpass $R$. Its first part is equal to $I$ and we seek now for the partition of $N-I$ of which the smallest part is at least $I$ and having the rank $R-\sum_{\mathrm{J}=1, \ldots, \mathrm{I}} P(N, \eta)$. 


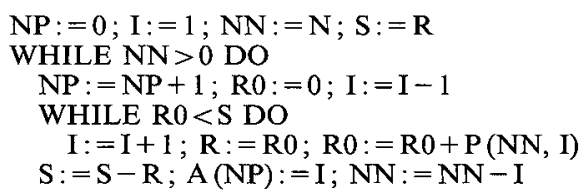

The number of arrays $T(A / B)$ is a weighted sum of the quantities $T\left(A^{\prime} / B^{\prime}\right)$ that we must compute, with the same principle of decomposition. Rather than to program a recursive function to compute many times the same thing, we store into an array $F$ the following values:

$\mathrm{F}(., 1):=N N$, quantity of which $A$ and $B$ are partitions;

$F(., 2):=R A$, rank of $A$ in the set of all the partitions of $N N$;

$F(., 3):=R B$, rank of $B$ in the set of all the partitions of $N N$;

$F(., 4):=$ weight of this couple of partitions.

The main step of the procedure to compute the number of arrays, is a decomposition of the partitions with ranks $R A$ et $R B$ if $N N>6$. Each row in $F$ generates others partitions of a smaller integer, since we remove the greatest part. These partitions are added into $F$. If $N N \leqq 6$, we do not perform this decomposition step, because we have provided the numbers of arrays that present any couple of partitions as sums of rows and columns.

The decomposition step is made with the following operations that we perform until $F$ is completely examined.

- We build $A$, partition of $N N$ with rank $R A$;

- We build $B$, partition of $N N$ with rank $R B$;

- If the greatest part of $A$ is greater than the greatest part of $B$, we exchange $A$ and $B$.

Let $A M$ and $B M$ be the greatest parts of $A$ and $B$, and $Q$ their weight.

- we remove from $B$ its greatest part and compute the rank $R$ of this partition of $N N-B M$;

- we enumerate the compositions of $B M$ bounded with $(D(I), I=1, \ldots, A M)$, where $D(I)$ is the number of parts in $A$ equal to I;

- for each composition $(C(I), I=1, \ldots, A M)$

- we compute the partition $P(I), I=1, \ldots, N P)$ obtained after subtracting from $A$, one unity to $C(I)$ parts equal to $\mathrm{I}$;

- we compute the rank $S$ of this partition;

- we compute the number $C O$ of ways to do these subtractions;

- we extend $F$ with a new row containing $N N-B M, R, S$, $C O, Q$. 
When the decomposition step is finished, we just have to add the products of the weights of the couple of partitions of integers $N N<6$ by the number of arrays that have these partitions as the sums of their rows and columns.

Exemple 3. $-A=(2233), B=(2233)$

\begin{tabular}{|c|c|c|c|c|c|c|c|}
\hline$C$ & $N N$ & $R A$ & $D$ & $R B$ & $B$ & $\mathrm{CO}$ & $Q$ \\
\hline & 10 & 33 & $\left(\begin{array}{llll}2 & 2 & 3 & 3\end{array}\right)=\left[\begin{array}{llll}0 & 2 & 2\end{array}\right]$ & 33 & (2233) & & 1 \\
\hline$\left[\begin{array}{lll}0 & 2 & 1\end{array}\right]$ & 7 & 6 & $\left(\begin{array}{llll}1 & 1 & 2 & 3\end{array}\right)=\left[\begin{array}{lll}2 & 1 & 1\end{array}\right]$ & 12 & (223) & 2 & 2 \\
\hline$\left[\begin{array}{lll}0 & 1 & 2\end{array}\right]$ & 7 & 8 & $\left(\begin{array}{lll}1 & 2 & 22\end{array}\right)=\left[\begin{array}{lll}1 & 3\end{array}\right]$ & 12 & (223) & 2 & 2 \\
\hline$\left[\begin{array}{lll}2 & 1 & 0\end{array}\right]$ & 4 & 3 & (13) & 4 & $(22)$ & impossib & \\
\hline$\left[\begin{array}{lll}2 & 0 & 1\end{array}\right]$ & 4 & 4 & (22) & 4 & (22) & 1 & 2 \\
\hline$\left[\begin{array}{lll}1 & 1 & 1\end{array}\right]$ & 4 & 2 & $\left(\begin{array}{lll}1 & 12 & 2\end{array}\right.$ & 4 & (22) & 2 & 4 \\
\hline [1 2] & 4 & 2 & $\left(\begin{array}{lll}1 & 1 & 2\end{array}\right)$ & 4 & (22) & 3 & 6 \\
\hline [03] & 4 & 1 & $\left(\begin{array}{llll}1 & 1 & 1 & 1\end{array}\right)$ & 4 & (22) & 1 & 2 \\
\hline
\end{tabular}

Finaly we get:

and $T(A / B)=34$.

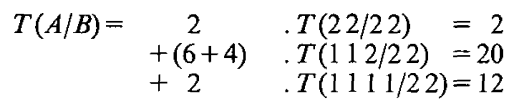

\section{CONSTRUCTION OF THE ARRAY WITH RANK $R$}

\subsection{Ordering $0 / 1$ arrays with fixed sums of rows and columns}

First we define a lexicographical order for boolean vectors with 1 before 0 . For vectors with 4 components of which 2 are equal to 1 , we get:

$$
\left(\begin{array}{llll}
1 & 1 & 0 & 0
\end{array}\right)<\left(\begin{array}{llll}
1 & 0 & 1 & 0
\end{array}\right)<\left(\begin{array}{llll}
1 & 0 & 0 & 1
\end{array}\right)<\left(\begin{array}{lll}
0 & 1 & 10
\end{array}\right)<\left(\begin{array}{llll}
0 & 1 & 0 & 1
\end{array}\right)<\left(\begin{array}{llll}
0 & 0 & 1 & 1
\end{array}\right) \text {. }
$$

Let $T$ be a $0 / 1$ array with $N r$ rows $R_{1}, \ldots, R_{N r}$. Each row is a vector with $N c$ components and $T$ another array with the same sums of rows and columns:

$$
T=\left(R_{1}, \ldots, R_{N r}\right), \quad T=\left(R_{1}^{\prime}, \ldots, R_{N r}^{\prime}\right)
$$

We place $T$ before $T$ if and only if $\exists k \mid \forall i<k R_{i}=R_{i}^{\prime}$ et $R_{k}<R_{k}^{\prime}$.

For this order, the arrays associated with the partitions (22 211 ) and (222) are successively:

$\begin{array}{llll}110 & 110 & 110 & 110 \\ 110 & 101 & 101 & 011 \\ 001 & 010 & 001 & 100 \\ 001 & 001 & 010 & 001, \text { etc. }\end{array}$

vol. $24, \mathrm{n}^{\circ} 1,1990$ 


\section{2. $R$ ranked array}

To build the array with rank $R$, we compute its rows according to increasing index, and apply the enumeration method proposed in [2]. Just as for the partition of given rank, we count the number of arrays of which the first row is as small as possible, until we reach or overpass $\mathbf{R}$.

We enumerate boolean vectors with $N c$ components of which $a_{1}$ are equal to 1 , in the previous order. To each vector $X=\left(x_{1}, x_{2}, \ldots, x_{N c}\right)$ corresponds arrays that have $X$ as first row. These arrays must have $\left(a_{2}, \ldots, a_{N r}\right)$ as sums of the other rows, and $\left(\left(b_{i}-x_{i}\right), i=1, \ldots, N c\right)$ as sums of columns. They are counted by the function defined above. Thus we just add these quantities until we reach or overpass $R$.

Then the first row is $X$ and we seek now for a $0 / 1$ array having $\left(a_{2}, \ldots, a_{N r}\right)$ as sums of rows, $\left(\left(b_{i}-x_{i}\right), i=1, \ldots, N c\right)$ as sums of columns and $R^{\prime}$ as rank, with:

$R^{\prime}=R-$ the number of arrays having a first row before $X$.

\section{Exemple 4:}

We build the 10-th array corresponding to partitions (2211) and (222).

There are $T\left(\begin{array}{llll}2 & 1 & 1 / 1 & 1\end{array}\right)=5$ of which the first row is $\left(\begin{array}{lll}1 & 1 & 0\end{array}\right)$

$$
T(211 / 121)=5
$$

So the first row is (101) and we seek for the 5-th corresponding to the partitions (2 111$)$ and (1 21 ).

There are $T(11 / 011)=2$ of which the first row is $(110)$

$$
\begin{aligned}
& T(11 / 020)=1 \\
& T(11 / 110)=2
\end{aligned}
$$

The second row is $\left(\begin{array}{lll}0 & 1 & 1\end{array}\right)$ and we seek the 2-nd corresponding to the partitions (1 11$)$ et $\left(\begin{array}{lll}1 & 1 & 0\end{array}\right)$.

There are $T(1 / 010)=1$ of which the first row is $(100)$

$$
T(1 / 100)=1
$$

the third row is $(010)$ and the last one is the remaining sums of the columns. The 10-th array is: 


\subsection{Algorithm}

To apply this algorithm we must enumerate the subsets with $a_{i}$ elements in a set with $N c$ elements. We borrow this classical algorithm to Nijenhuis et Wilf [3]. But all these subsets are not suitable, since some columns can be saturated, and the general algorithms is:

Let $(D A(I), I=1, N r)$ and $(D B(I), I=1, N c)$ be the specified sums of rows and columns, and $R$ the rank of the searched narray.

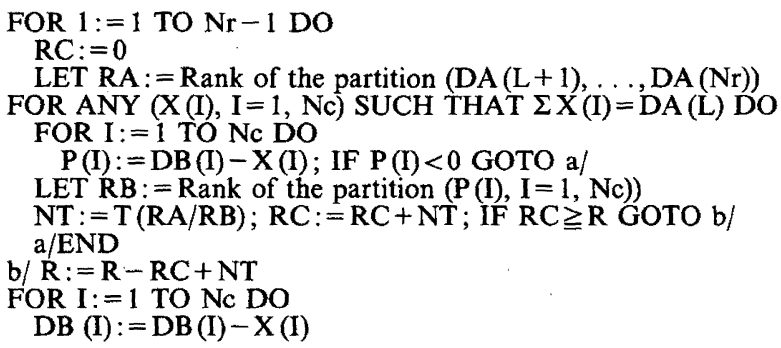

\subsection{Complexity and conclusions}

The advantage announced in the introduction - succeed at the first shot - is counter balanced by a high complexity. Even if we admit that all the necessary countings are tabulated, we must, in the worst case where the wanted array is the last one, enumerate for each row all the subsets with cardinality $a_{i}$ in a set with $N c$ elements. The worst case will be reached when $a_{i}=N c / 2$. Then, there are $(N c \backslash N c / 2)$ subsets. Thus we have a complexity $O\left(N r .2^{N c-1}\right)$.

Therefore, the performance of this algorithm can be compared with N. C. Wormald's only in his difficult cases, that is when the searched array is practically full of ones. In this case, my algorithm is faster than his, but we can find very quickly, with the Wormald method, the complement of the searched array. More over, his algorithm requires only a few instructions.

It remains that we can compute tables for the number of bipartite graphs with specified degrees, as we show in the appendix, and select them at random following any distribution law, since it is the rank that is selected at random.

\section{REFERENCES}

[1] Cl. Berge, Principes de Combinatoire, Dunod, Paris, 1968.

[2] A. Guénoche, Énumération de classes de permutations, RAIRO Recherche Opérationnelle, Vol. 13, No. 4, 1979, pp. 379-390.

[3] H. Nisenhuis \& H. Wilf, Combinatorial Algorithms, Acad. Press, New York, 1978.

[4] N. C. Wormatd, Generating random regular graphs, Journal of Algorithms, Vol. 5, No. 2, 1984, pp. 247-280. 


\section{Appendix: Tables}

The degree sequences of vertices of each part is a partition of an integer $N$ equal to the number of egdes. The number of bipartite graphs with a couple of partitions is given in the following tables whose entries are the partitions of $3 \leqq N \leqq 10$. They are labelled in decreasing number of parts order, then in lexicographical order. For each table, the column order is the same as the row one, and for instance, the number of bipartite graphs with degree sequences (1 111112 ) and (123) can be read in the table of partitions of 6 ; These sequences have ranks 2 and 6 , so there are 22 graphs with these degrees.

$$
\begin{aligned}
& \text { Partitions of } 3 \\
& \begin{array}{l}
1: 111: 6 \\
2: 12: 31 \\
3: 3: 100
\end{array}
\end{aligned}
$$

Partitions of 4

$1: 1111: 24$

2:112: 125

$3: 13: 410$

$4: 22: 6201$

$5: 4: 10000$

\section{Partitions of 5}

$1: 11111: 120$

2:1112: 6027

$3: 113: 2071$

$4: 122: 301225$

$5: 14: 51000$

$6: 23: 1030100$

$7: 5: 1000000$

Partitions of 6 .

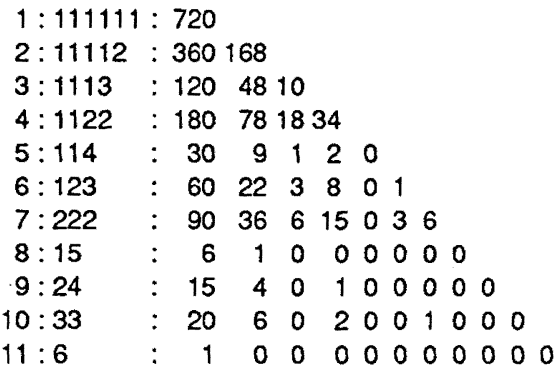

Recherche opérationnelle/Operations Research 
Partitions of 7

$1: 1111111: 5040$

$2: 111112: 25201200$

$3: 11113 \quad: \quad 840 \quad 360 \quad 88$

$4: 11122: 1260570150258$

$5: 1114 \quad: \quad \begin{array}{lllll}210 & 75 & 13 & 24 & 1\end{array}$

$6: 1123 \quad: \quad 420 \quad 170 \quad 34 \quad 68 \quad 313$

$7: 1222 \quad: \quad 630 \quad 270 \quad 60117 \quad 62751$

$8: 115: \begin{array}{rllllllll}42 & 11 & 1 & 2 & 0 & 0 & 0 & 0\end{array}$

$9: 124 \quad: \quad \begin{array}{lllllllll}105 & 35 & 4 & 11 & 0 & 1 & 3 & 0 & 0\end{array}$

$10: 133 \quad: \quad 140 \quad 50 \quad 6 \quad 18 \quad 0 \quad 2 \quad 7 \quad 0000$

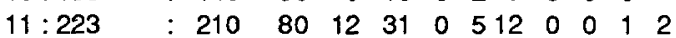

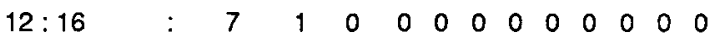

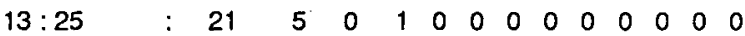

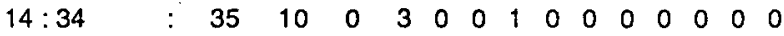

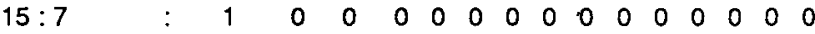

Partitions of 8

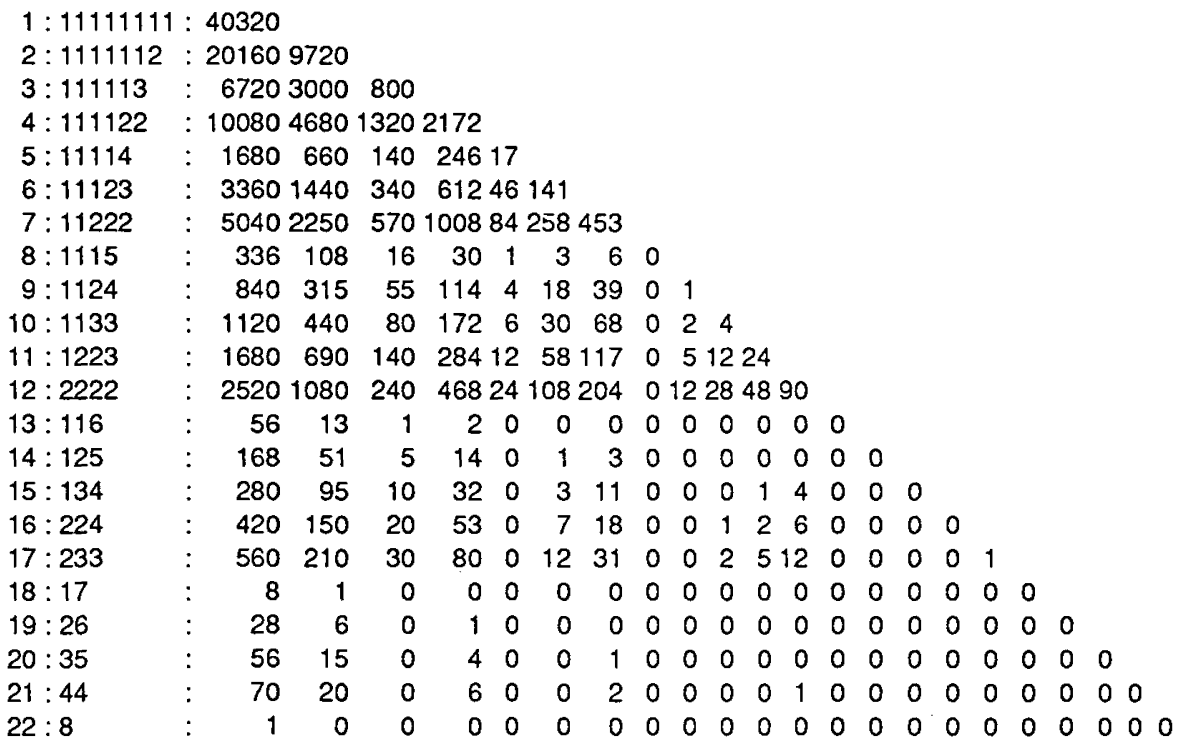

vol. $24, \mathrm{n}^{\circ} 1,1990$ 
Partitions of 9

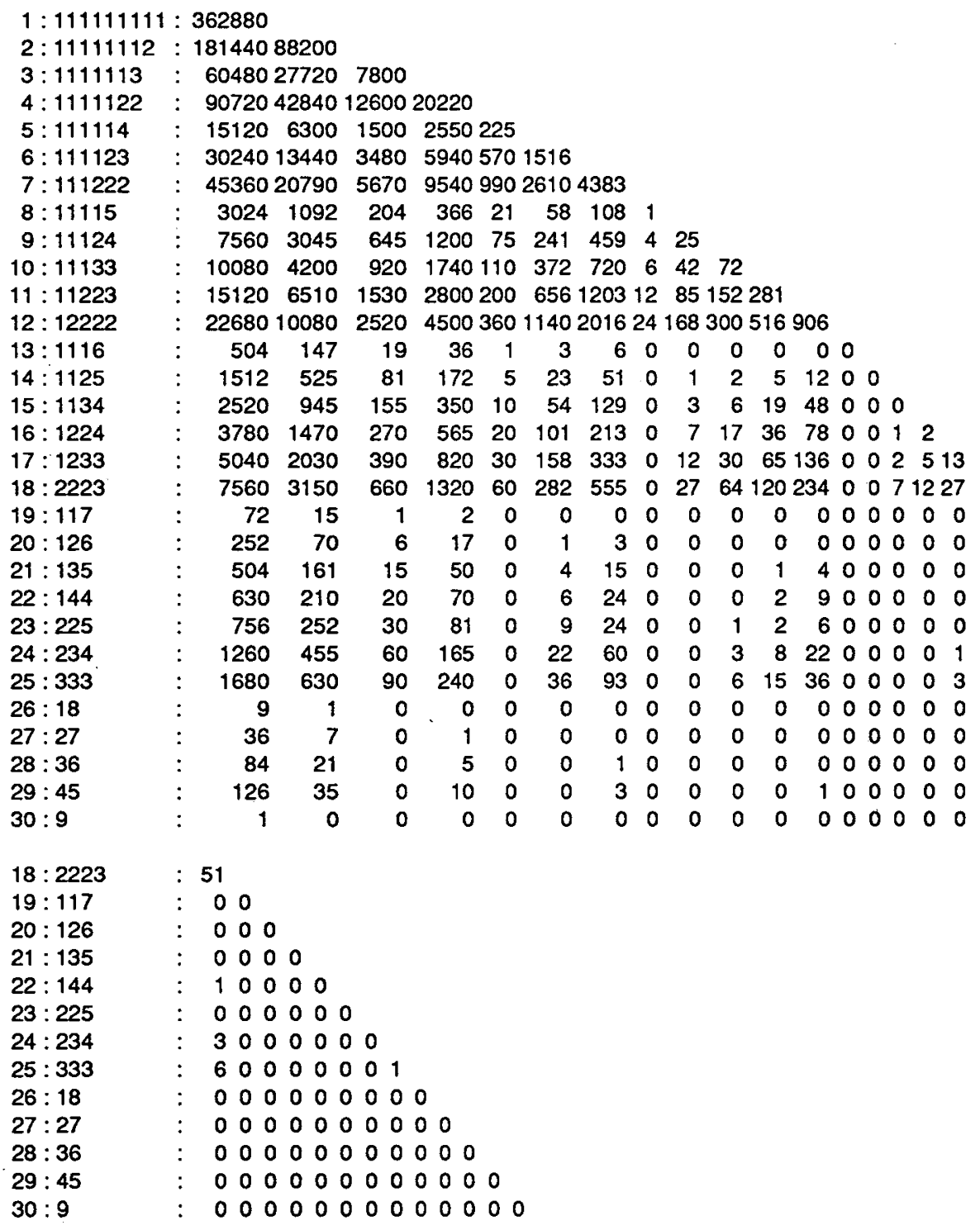


Partitions of 10

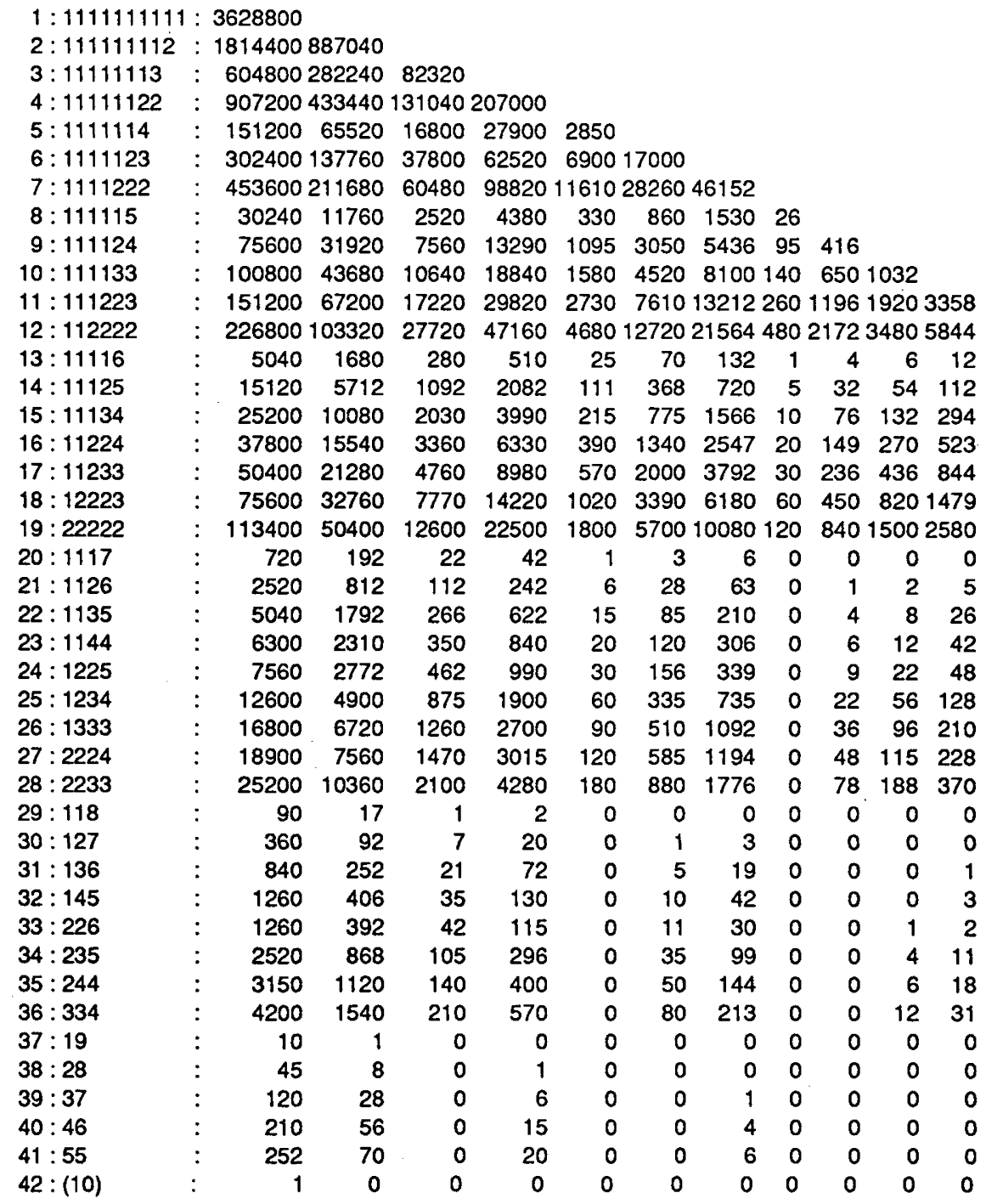




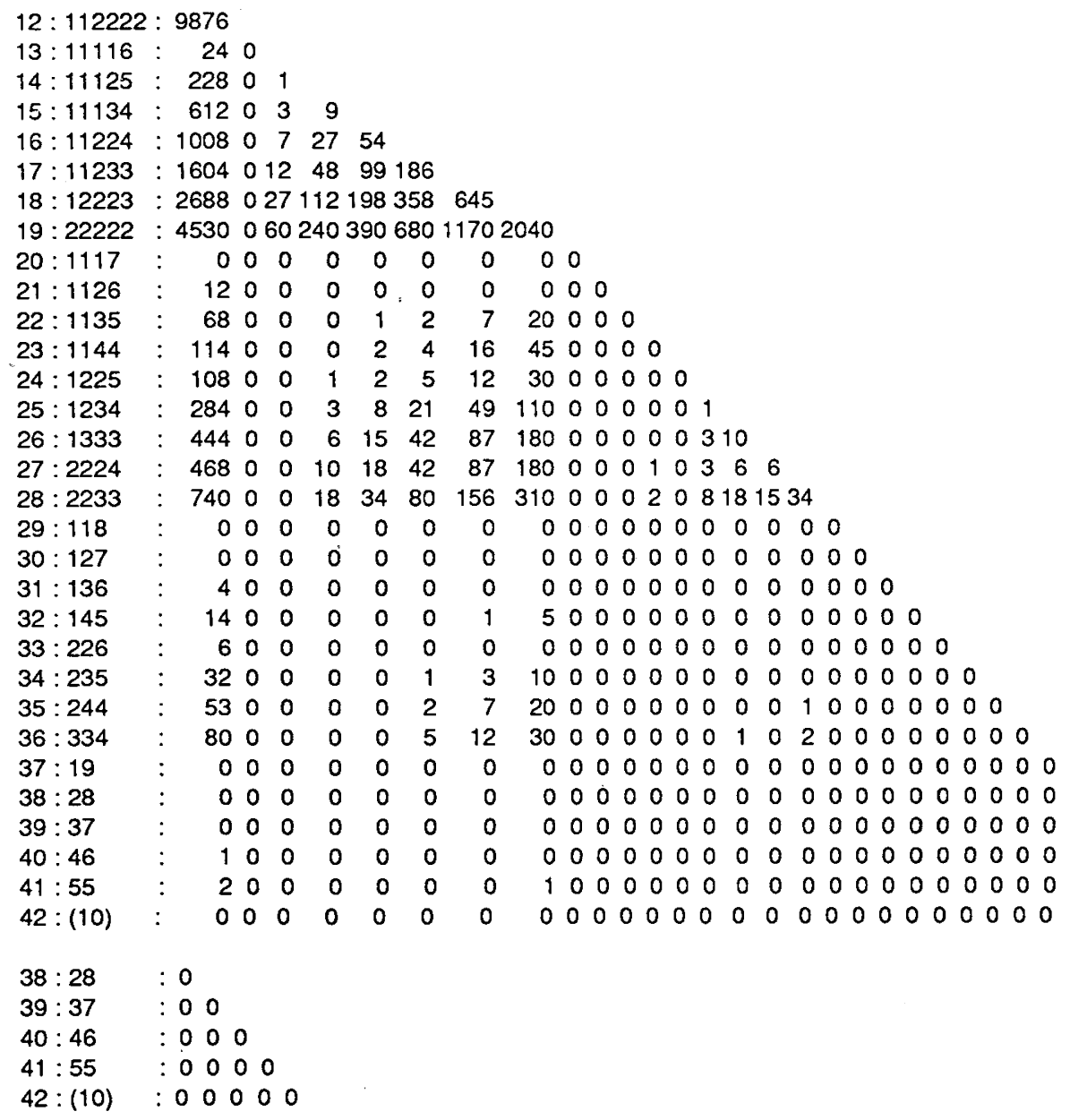

